\title{
Control of Dispersed Generation to Regulate Distribution and Support Transmission Voltages
}

\author{
Gustavo Valverde \\ Department of Electrical Engineering \\ and Computer Science \\ University of Liège \\ Liège, Belgium \\ gvalverde@ieee.org
}

\author{
Thierry Van Cutsem \\ Fund for Scientific Research (FNRS) at \\ Department of Electrical Engineering \\ and Computer Science \\ University of Liège \\ Liège, Belgium \\ t.vancutsem@ulg.ac.be
}

\begin{abstract}
This paper presents an on-line centralized controller based on Model Predictive Control concepts to regulate distribution network voltages, and possibly support transmission network. It computes and applies a sequence of changes of the distributed generators power outputs to progressively meet a set of operation constraints at distribution, and possibly transmission level. An 11-kV, 75-bus test system with 22 distributed generators is used to illustrate the performance of the proposed controller when transmission system support is requested. Comparisons with single-step open-loop control are also provided.

Index Terms-Voltage control, model predictive control, distributed generation, transmission system support.
\end{abstract}

\section{INTRODUCTION}

The integration of dispersed generation in distribution networks has opened new opportunities to optimize the network operation, e.g., power losses minimization and voltage regulation [1], [2]. Moreover, it is quite plausible that active distribution networks will be requested to support the transmission network, either by helping to maintain certain voltage levels at the connection point or by operating with reactive power in a specified range of values. For instance, the European Network of Transmission System Operators for Electricity (ENTSOE) is defining a set of common requirements at the interface between transmission and distribution networks. Among these requirements, the reactive power should not be outside a range that depends on the import or export capability [3]. Based on this, distribution networks will have to meet their own voltage constraints while trying to satisfy the reactive power demands of the transmission grid.

In Ref. [4] a linear optimization problem is solved to maximize the Distributed Generator (DG) reactive power output, and reduce the reactive power import from the transmission grid. In [5], the authors also solve an optimization problem to minimize the reactive power import/export. The solution provides a reference voltage for the transformer Load Tap Changer (LTC) and the optimal power factor of the DG units.

References [4]-[5] present passive control strategies because they determine fixed set-points for the LTC and DGs for a season or year. Although the system operator could try to meet these set-points, the uncertainty in demand and generation plus unexpected disturbances will require adjusting those set-points to correct for bus voltage and reactive power transfer violations. In this sense, corrective control schemes fed by real-time measurements are necessary to meet all the constraints. For example, a corrective voltage control scheme that minimizes the reactive power deviations of DGs subject to network constraints is presented in [6]. The authors use sensitivity analysis to estimate the change of controlled bus voltages with changes of reactive power of DGs.

In [7], the authors propose a multi-objective optimizer that adjusts the DG outputs to minimize voltage and control variable deviations with respect to a day-ahead scheduler, subject to network operation constraints. Reference [8] presents a 24-hour multi-objective optimization problem for optimal operation of micro-grids. Here, the controller calculates the outputs of DGs, controllable loads and storage devices to minimize network losses, generation costs and gas emissions. In order to account for the uncertainty of renewable energy sources and load forecasts, the control scheme updates the 24-h optimization problem given the latest estimate of bus voltages and the latest forecast of weather and loads.

The work in [9] uses the principle of the French secondary voltage control and applies it to distribution networks. Firstly, the voltage reference of pilot bus voltages is computed to minimize the network losses. Then, the controller optimally corrects LTC and DG set-points to regulate pilot bus voltages.

This paper presents a new short-term corrective control scheme to maintain distribution network voltages within prespecified limits. The proposed centralized controller is inspired of Model Predictive Control (MPC) [10]; thus, it takes its decisions based on real-time measurements. MPC is attractive for its ability to smoothly drive the system from the current to the target state, take into account its near-future evolution, and compensate for modelling inaccuracies, especially those affecting the behaviour of loads located near the DG units. The method was detailed in [11]. In this paper, the formulation is extended to account for Transmission System Operator (TSO) requests regarding either the voltage or the reactive power exchange at the connection point.

This paper is organized as follows. The control algorithm is presented in Section II, while Section III reports on numerical simulations. A comparison with single-step open-loop control 


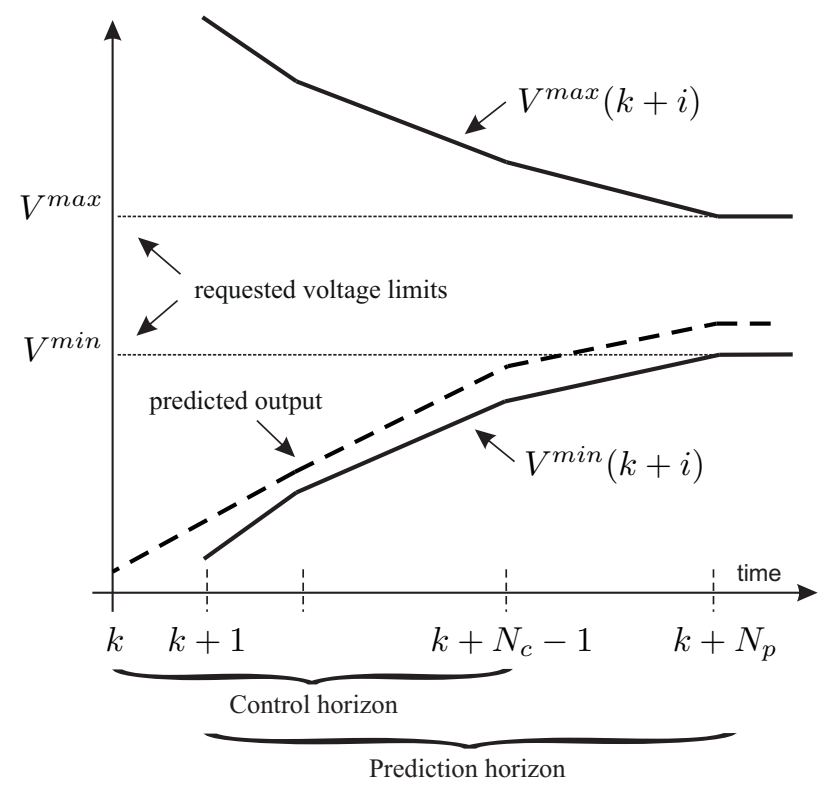

Figure 1. Basic concept of MPC applied to voltage control

is offered in Section IV. Conclusion and perspectives are presented in Section V.

\section{Control of Distribution Network Voltages}

The proposed control aims at progressively bringing the distribution network voltages in a pre-specified range of values. This is outlined in Fig. 1, relative to one of the controlled voltages. At time $k$, the MPC-based control uses an internal model to predict the behavior of the system over a future prediction interval with $N_{p}$ discrete steps, and computes an optimal sequence of $N_{c}$ control actions, where $N_{c} \leq N_{p}$ [10]. The solid lines in Fig. 1 show the constraints progressively imposed on voltage, in order to bring the latter inside the requested limits at the end of the prediction interval [11].

Thus, at time step $k$, using the latest available measurements, the controller determines the sequence of control variable changes $\Delta \boldsymbol{u}(k), \Delta \boldsymbol{u}(k+1), \ldots, \Delta \boldsymbol{u}\left(k+N_{c}-1\right)$ that optimizes a multi-step objective with constraints. The changes are between consecutive values, i.e.

$$
\Delta \boldsymbol{u}(k)=\boldsymbol{u}(k)-\boldsymbol{u}(k-1)
$$

According to MPC principle, only $\Delta \boldsymbol{u}(k)$ is applied. At the next time step $k+1$, based on the newly available measurements, the whole control change sequence is recomputed and, again, only the first component of the sequence is applied.

In order to correct the distribution voltages, the controller may use the voltage set-point $V_{t a p}$ of the transformer LTC and the active and reactive power outputs of the DGs, denoted $\boldsymbol{P}_{g}$ and $\boldsymbol{Q}_{g}$. The change of control variables at time step $k$ are:

$$
\Delta \boldsymbol{u}(k)=\left[\Delta \boldsymbol{P}_{g}(k)^{T}, \Delta \boldsymbol{Q}_{g}(k)^{T}, \Delta V_{\text {tap }}(k)\right]^{T}
$$

where ${ }^{T}$ denotes array transposition.

The active power output of DGs are considered expensive control variables and they should not be changed unless emergency conditions are faced. On the contrary, the LTC setpoint and the reactive power output of DGs are assumed cheap control variables [11].

The multi-step objective involves all control changes in the control horizon. More precisely, the following quadratic objective is considered at time $k$ :

$$
\min _{\Delta \boldsymbol{u}, \varepsilon} \sum_{i=0}^{N_{c}-1}\|\Delta \boldsymbol{u}(k+i)\|_{\boldsymbol{R}}^{2}+\|\varepsilon\|_{\boldsymbol{S}}^{2}
$$

where the squared control changes are considered to more evenly distribute the effort among the available controls, and $\boldsymbol{R}$ is a weight matrix used to force control priorities. The slack variables $\varepsilon=\left[\varepsilon_{1}, \varepsilon_{2}, \varepsilon_{3}, \varepsilon_{4}\right]^{T}$ are used when some of the constraints detailed hereafter make the optimization problem infeasible. These variables are heavily penalized using the weight matrix $S$, to keep them at zero whenever possible.

The minimization is subjected to the following constraints:

- Control variables constraints: for $i=0,1, \ldots, N_{c}-1$ :

$$
\begin{aligned}
\boldsymbol{u}^{\text {min }} & \leq \boldsymbol{u}(k+i) \leq \boldsymbol{u}^{\text {max }} \\
\Delta \boldsymbol{u}^{\text {min }} & \leq \Delta \boldsymbol{u}(k+i) \leq \Delta \boldsymbol{u}^{\text {max }}
\end{aligned}
$$

The limits $\boldsymbol{u}^{\min }$ and $\boldsymbol{u}^{\max }$ are defined based on the capabilities of the equipment (LTC or distributed generators), while $\Delta \boldsymbol{u}^{\min }$ and $\Delta \boldsymbol{u}^{\max }$ are associated with the permitted ramping rate of equipment.

- Distribution voltage constraints: for $i=1,2, \ldots, N_{p}$ :

$$
\begin{gathered}
-\varepsilon_{1} \mathbf{1}+\mathbf{V}^{\min }(k+i) \leq \boldsymbol{V}(k+i \mid k) \leq \boldsymbol{V}^{\max }(k+i)+\varepsilon_{2} \mathbf{1} \\
\boldsymbol{V}(k+i \mid k)=\boldsymbol{V}(k+i-1 \mid k)+\frac{\partial \boldsymbol{V}}{\partial \boldsymbol{u}} \Delta \boldsymbol{u}(k+i-1) \\
+\frac{\partial \boldsymbol{V}}{\partial V_{\text {tap }}} \Delta V_{d} \gamma(k+i)
\end{gathered}
$$

Here, $\boldsymbol{1}$ denotes a unitary vector, $\boldsymbol{V}^{\min }(k+i)$ and $\boldsymbol{V}^{\max }(k+i)$ are the voltage limits at the $i$-th prediction step, as shown in Fig. 1, and $\boldsymbol{V}(k+i \mid k)$ is the vector of predicted bus voltages given the measurements at time $k . \boldsymbol{V}(k \mid k)$ is set to the real-time measurements collected at time step $k$. A linear approximation is used to predict voltages in (2e), relying on the sensitivities of voltages to control variables $\frac{\partial V}{\partial u}$, and to the LTC voltage set-point $\frac{\partial V}{\partial V_{t a p}}$. Finally, $\Delta V_{d}$ is the assumed variation of the LTC-controlled voltage for a single tap change, whereas $\gamma$ is a binary variable equal to one for the instants when tap changes are predicted, and zero otherwise [11].

The sensitivity of bus voltages to power injections can be obtained from the inverse of the Jacobian matrix extracted from a power flow calculation [7]. The sensitivities $\frac{\partial V}{\partial V_{\text {tap }}}$ can be approximated by computing the ratio of variations of the monitored bus voltages to the LTC's controlled voltage due to a tap change. All sensitivities are computed off-line and infrequently updated. 
- Transmission operation constraints: for $i=1,2, \ldots, N_{p}$ :

$$
\begin{gathered}
-\varepsilon_{3}+y^{\min }(k+i) \leq y(k+i \mid k) \leq y^{\max }(k+i)+\varepsilon_{4} \\
y(k+i \mid k)=y(k+i-1 \mid k)+\frac{\partial y}{\partial \boldsymbol{u}} \Delta \boldsymbol{u}(k+i-1) \\
+\frac{\partial y}{\partial V_{\text {tap }}} \Delta V_{d} \gamma(k+i)
\end{gathered}
$$

where $y$ is either the bus voltage or the reactive power exchanged with the transmission system at the connection point. $y(k+i \mid k)$ is the predicted value given the measurements at time $k$. The sensitivities involved in $(2 \mathrm{~g})$ are evaluated similarly to those in (2e). $y(k \mid k)$ is set to the real-time measurements collected at time step $k$.

\section{Simulation Results}

\section{A. Test system and simulation conditions}

The proposed controller has been extensively tested through simulations of a radial 75 -bus, $11-\mathrm{kV}$ distribution network hosting 22 DG units. A first set of results obtained with this system is available in [11]. In this paper, the emphasis is on the use of the controller to support transmission voltages.

The network topology is shown in Fig. 2. The line parameters and operation point data can be found in [12]. The distribution system is connected to an external grid, modeled as a Thévenin equivalent, through a $33 / 11 \mathrm{kV}$ transformer equipped with LTC. The network consists of eight feeders all directly connected to the main transformer. It serves 38 loads modeled as constant current for active power and constant impedance for reactive power, and 15 loads represented by equivalent induction motors.

Among the 22 DG units, 13 are 3-MVA synchronous generators and the remaining are 3.3-MVA Doubly Fed Induction Generators (DFIGs). Each synchronous generator is controlled by an automatic voltage regulator with an inner control loop to regulate the terminal voltage in response to fast changes, and an outer PI control loop to adjust the reactive power to the set-point demanded by the centralized controller. Similarly, the DFIGs operate in reactive power control mode to meet the power output requested by the proposed controller.

The simulations were carried out with RAMSES, a dynamic simulator developed at the Univ. of Liège [13]. A discretetime controller was implemented, solving the Quadratic Programming problem (2a)- $(2 \mathrm{~g})$ with the VE17AD library from Harwell [14]. The controller requests changes of the DG reactive power outputs every $10 \mathrm{~s}$, using $N_{c}=N_{p}=3$, unless future LTC actions require increasing $N_{p}$ [11]. Thus, although the proposed control acts in the range of a few minutes, its behaviour was checked in the presence of transients stemming from DGs and loads.

The following measurements were simulated: terminal voltage, active and reactive power of each DG, voltage at three load buses (see Fig. 2), voltage at both ends of the transformer and reactive power exchange with external grid (reactive flow leaving bus 1000 and entering the transformer). The "measured" values were obtained by adding to the outputs of time simulation a white Gaussian noise restricted to $\pm 0.01 \mathrm{pu}$

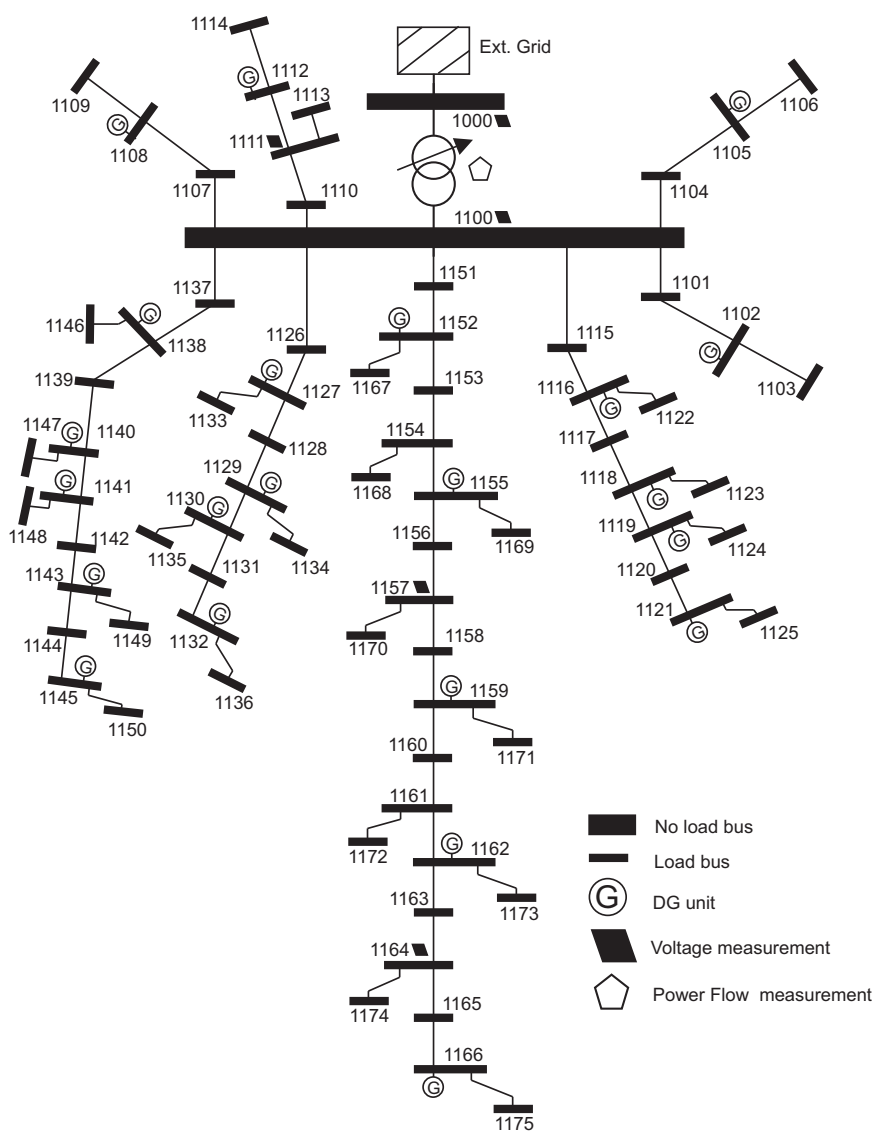

Figure 2. Network topology and measurement location

for voltages, $\pm 1 \%$ of the actual reactive power exchange, and $\pm 1 \%$ of the respective DG maximum output for their active and reactive powers. Measurements were collected some time after control actions were applied, to avoid making decisions based on measurements taken during transients. In addition, the noise is filtered by using the average of 11 snapshots received in a time window of two seconds.

To demonstrate the robustness with respect to model inaccuracies, the sensitivity values have been calculated considering:

AS: the exact short-circuit level of 200 MVA (Thévenin reactance of $0.50 \mathrm{pu}$ ) for the external network along with the exact model of loads. The resulting accurate sensitivity matrices are referred to as $A S$;

IS1: an underestimated Thévenin reactance of $0.25 \mathrm{pu}$ and constant power loads, yielding inexact sensitivity matrices, denoted ISI;

IS2: an overestimated Thévenin reactance of $0.75 \mathrm{pu}$ and constant power loads, yielding sensitivity matrices $I S 2$.

\section{B. Correction of Distribution and External Voltages}

It is assumed that the distribution voltages must remain within [1.000 1.025] pu, and the external grid requests the distribution network to maintain v1000, the voltage at bus 1000 , within [0.970 1.030] pu. The reactive power of the DGs are the only control variables used to correct the voltages. 


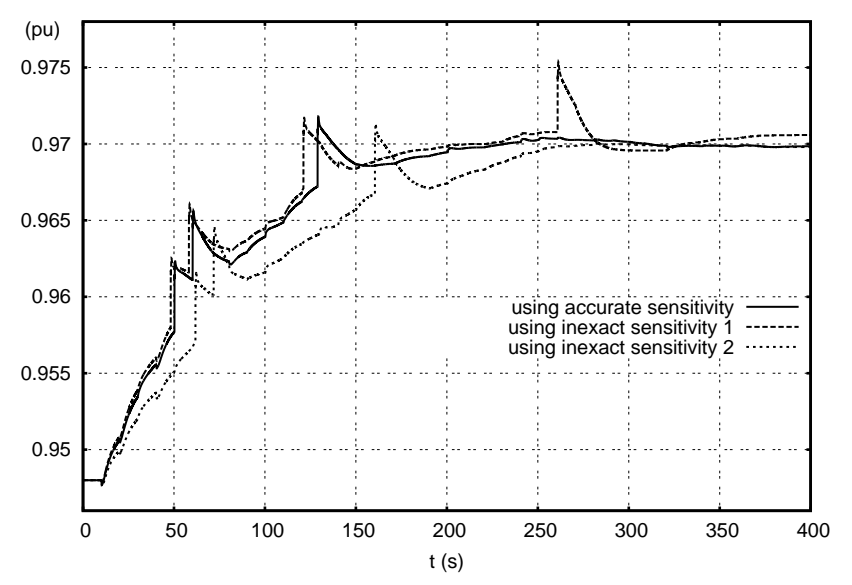

Figure 3. External bus voltage correction

Therefore, the LTC voltage set-point is not used as control variable, but the LTC is allowed to perform its local control to maintain the distribution voltage v1100 within its [0.990 1.010] pu dead-band. This LTC has an initial operation delay of $20 \mathrm{~s}$ and $10 \mathrm{~s}$ for subsequent tap movements, if required. These tap movements are anticipated by the controller and the effect on voltages is considered in (2e).

Figure 3 presents the correction of the external bus voltage v1000 by optimal changes of the DG reactive power outputs using the aforementioned sensitivity matrices. The changes of the reactive power outputs trigger at least three tap movements to maintain v1100 within the allowable dead-band, see Fig. 4.

The main difference between the plots in Fig. 3 is the time response of the controller. It is seen that $A S$ and ISI yield very similar system evolutions. However, an additional tap movement is triggered by the more "aggressive" control response with ISI, where the inaccuracy on the Thévenin reactance is partially compensated by the error on load models. With $I S 2$, the two types of errors add up. Here, the controller assumes that the voltages will respond more than in reality. In other words, its control changes have smaller effects than anticipated from the model. This is compensated by acting for a longer time, which explains why the system response is slower with $I S 2$ than with $A S$ or ISI.

Figure 4 presents the evolutions of the voltages at bus 1100 and three representative buses within the distribution network (namely 1127, 1145 and 1166) using the ISI matrices. Initially, the bus voltages $\mathrm{v} 1000$ and v1145 are outside the acceptable ranges of operation. Due to the corrections, some of the distribution network voltages go above $1.025 \mathrm{pu}$ at three different periods, as detailed in Fig. 4. Here, the controller temporarily relaxed the voltage limits in (2d), using $\varepsilon_{2}>0$. In addition, since v1100 goes outside the LTC voltage deadband, the LTC acts at $t=46,56,120$ and $260 \mathrm{~s}$. By doing so, the distribution voltages return to the permissible range of operation and the controller succeeds in correcting v1000 while keeping the distribution network voltages within limits.

Figure 5 presents the power outputs of some DGs. Note that

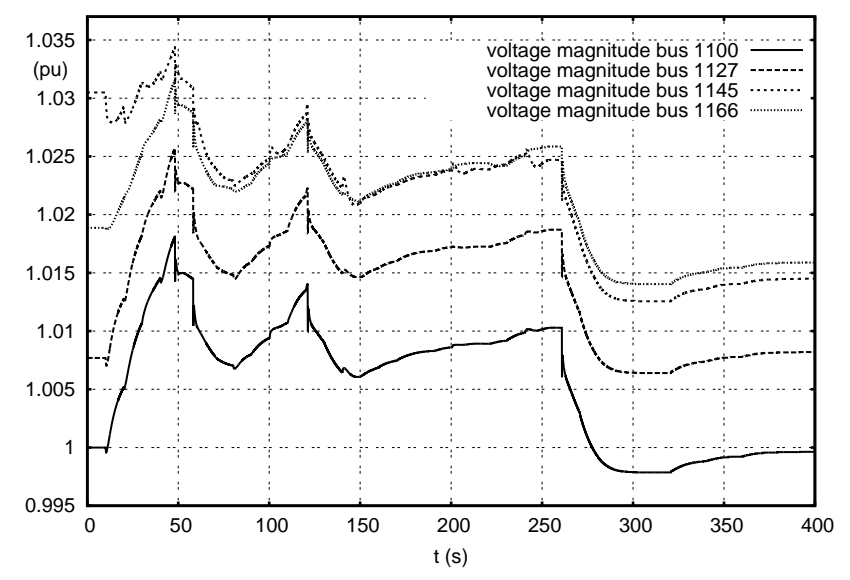

Figure 4. Distribution bus voltage corrections using ISI

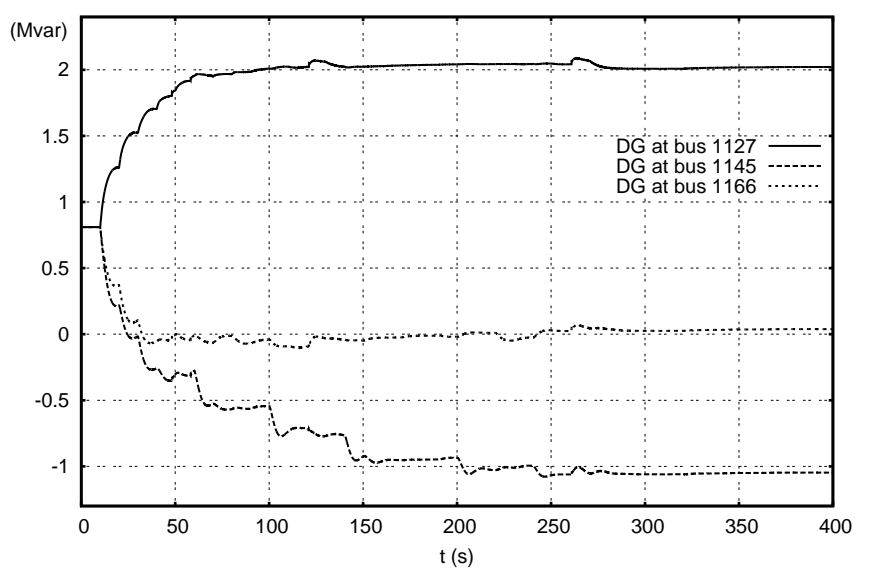

Figure 5. Reactive power outputs of some DGs using ISI

the DG closer to the main transformer (at bus 1127) provides more reactive power to correct $\mathrm{v} 1000$. On the contrary, the DGs at the end of the feeders reduce their reactive powers to avoid over voltages inside the distribution network.

\section{Correction of Reactive Power Exchange}

As explained in the Introduction, the external grid operator may request the distribution network to keep the reactive power transfer within a certain range. In this test case, the external grid operator requests the distribution network to improve the power factor at the connection point.

Initially, the distribution network imports 11.2 Mvar while all DG units operate at unity power factor. Then, the distribution network is requested to reduce the reactive power import to 5 Mvar or less, given that the active power export is $13 \mathrm{MW}$. In addition, the controller must keep the distribution voltages within [1.000 1.025] pu, and the LTC keeps v1100 within [0.995 1.015] pu.

Figure 6 presents the correction of the reactive power exchange from bus 1000 to 1100 using the three available sensitivity matrices. By applying changes of the DG reactive power outputs, the controller is able to reduce the reactive 


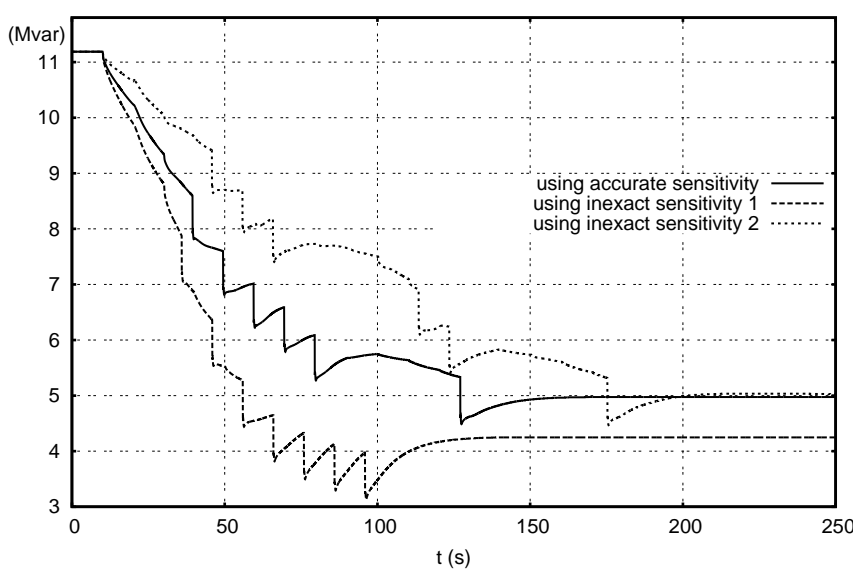

Figure 6. Correction of reactive power exchange

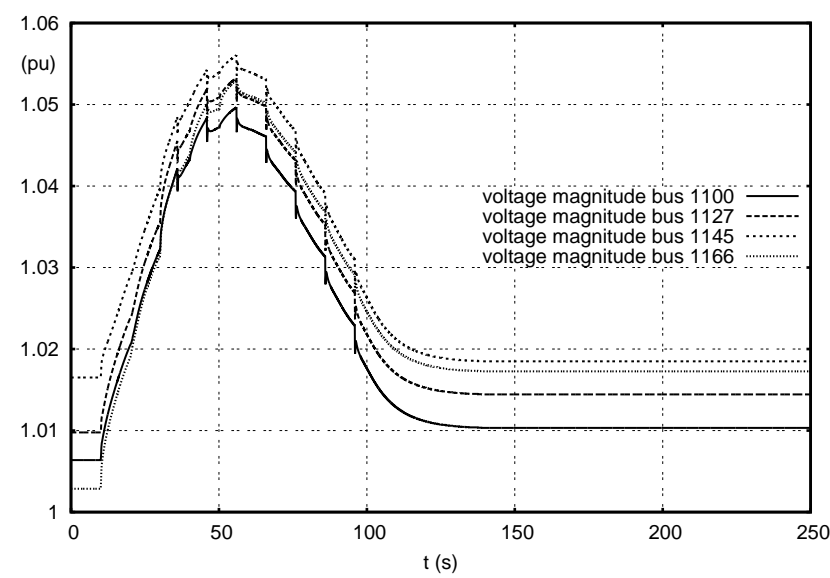

Figure 7. Distribution bus voltages evolution while controlling the reactive power exchange using $I S I$

power to 5 Mvar or less. In this test case, the controller acts faster when using the inexact sensitivities $I S 1$. When using $I S 2$ matrices, the controller predicts that the controlled variables can reach the desirable regions with little control efforts. This model inaccuracy makes the controller to correct the power transfer much later if compared to $A S$ and $I S 1$ matrices.

Figure 7 presents the evolution of the distribution bus voltages during the correction of the reactive power exchange, using ISI matrices. Due to the DG reactive power increase, the monitored bus voltages leave the allowable range of operation at $t=25 \mathrm{~s}$. Similarly, v1100 also violates the LTC dead-band, and this triggers seven consecutive tap movements starting at $t=35 \mathrm{~s}$. After these tap changes all voltages fall within the acceptable limits and the controller takes no further action. These tap movements were all anticipated through (2e) and $(2 \mathrm{~g})$; this allowed to request more reactive power from DG units as the controller predicted that the LTC would eventually correct the temporary over voltages.

\section{COMPARISON WITH SINGLE-STEP CONTROL}

\section{A. Formulation}

Comparisons were carried out between the proposed MPCbased and a single-step optimization-based control. The latter is found to be the most common formulation in centralized voltage control schemes, see for example [6], [7].

With the same type of objective and constraints, the singlestep optimization takes on the form:

$$
\min _{\Delta \boldsymbol{u}, \varepsilon}\|\Delta \boldsymbol{u}\|_{\boldsymbol{R}}^{2}+\|\varepsilon\|_{S}^{2}
$$

subject to:

$$
\boldsymbol{u}^{\min } \leq \boldsymbol{u} \leq \boldsymbol{u}^{\max }
$$

$$
\begin{aligned}
-\varepsilon_{1} \mathbf{1}+\mathbf{V}^{\text {min }} & \leq \boldsymbol{V}_{\text {meas }}+\frac{\partial \boldsymbol{V}}{\partial \boldsymbol{u}} \Delta \boldsymbol{u} \leq \boldsymbol{V}^{\text {max }}+\varepsilon_{2} \mathbf{1} \\
-\varepsilon_{3}+y^{\text {min }} & \leq y_{\text {meas }}+\frac{\partial y}{\partial \boldsymbol{u}} \Delta \boldsymbol{u} \leq y^{\text {max }}+\varepsilon_{4}
\end{aligned}
$$

where $\boldsymbol{V}_{\text {meas }}$ and $y_{\text {meas }}$ refer to measured values. With this formulation, the prediction capabilities of MPC are lost. Furthermore, all actions are imposed at once; hence, the smooth control changes achieved with the MPC-based formulation are replaced by abrupt control changes that could be detrimental for the distribution network. Finally, while MPC resembles closed-loop control, the single-step formulation is definitely open-loop. It results that model inaccuracies (e.g. of sensitivity matrices) will impact the control decisions, with no automatic way of correcting them.

\section{B. Simulation results}

The simulation results hereafter confirm this claim. They were obtained using the same initial conditions, sensitivity matrices and voltage limits as in the previous section.

Figure 8 presents the partial voltage correction of bus 1000 by applying a single correction at $t=10 \mathrm{~s}$. Only for $I S I$ the controller is able to correct v1000. On the other hand, only partial corrections were achieved when $A S$ and $I S 2$ were used.

The discrete events starting at around $t=37 \mathrm{~s}$ correspond to the LTC actions to maintain v1100 within its dead-band.

Under this control scheme, the controller requests large machine power corrections to try to recover the voltages at once. This is opposite to the proposed MPC approach which distributes the effort along the control horizon resulting in smooth and successful corrections of the controlled voltages.

In the last test case, the single-step control scheme is also used to correct the reactive power exchange at the connection point, as in Section III-C.

Figure 9 presents the unsuccessful correction of the reactive power flow when using formulation (3a) - (3d). Here, model inaccuracies have more impact on the performance of the controller. Irrespective of the sensitivity matrices, the singlestep control scheme is not enough to correct the flow to 5 Mvar or lower. This further highlights the importance of using closed-loop control schemes able to correct for initial yet insufficient control decisions. 


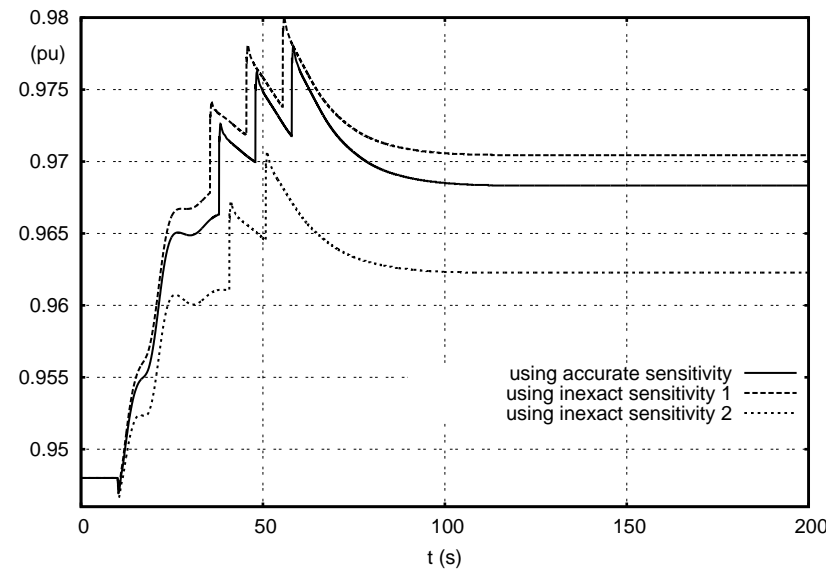

Figure 8. External bus voltage correction using single-step optimization

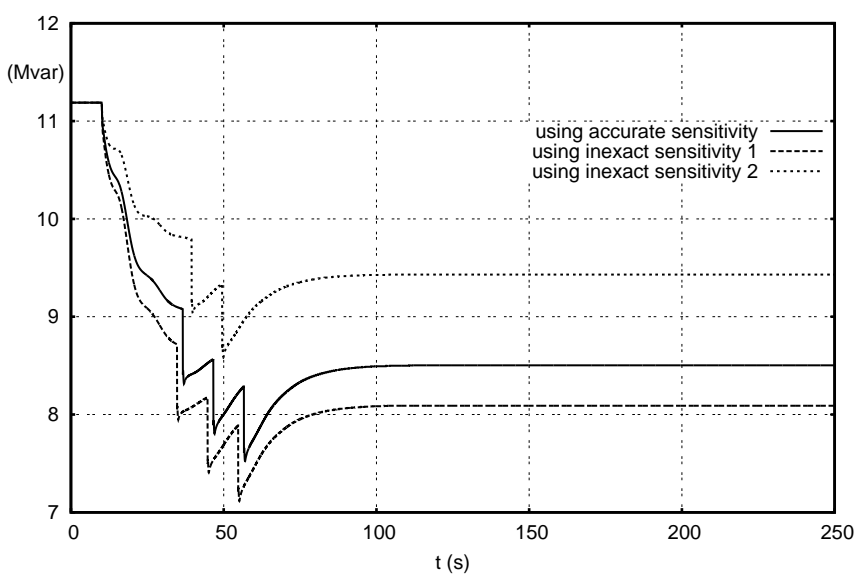

Figure 9. Reactive power exchange partial correction using single-step optimization

\section{CONCLUSION AND PERSPECTIVES}

An MPC-based control scheme for regulating distribution networks has been presented in this paper. The previous formulation has been extended to deal with transmission system, by including constraints on either the voltage or the reactive power exchange at the connection point.

The paper also illustrates the benefits with respect to a single-step optimization-based approach, as considered in most publications.

The robustness of the proposed controller was validated from simulations incorporating the fast dynamics of the controlled DG units. The results confirm that the proposed control can smoothly correct the controlled variables, even in the presence of significant model inaccuracies.

The test cases presented in this paper rely on a $11-\mathrm{kV}$ distribution network, with high penetration of distributed generation, connected to a rather weak $33-\mathrm{kV}$ sub-transmission network. The system was chosen so that changes of DG reactive power outputs can significantly affect the voltage at the connection point (i.e. the sub-transmission voltage was controllable by the DG units). In a stronger and stiffer transmission network, it is more likely that the TSO will request the reactive power transfer at the connection point to be within some limits (or, equivalently, a minimum power factor will be requested). However, the idea of controlling transmission voltages remains credible if many among the connected distribution systems share this effort.

This piece of work is one step further towards demonstrating how active distribution networks will be able to partially support transmission networks. Future work will concentrate on studying the interaction of various distribution networks participating in transmission voltage support during emergency conditions. For this, a large-scale model combining transmission and distribution systems will have to be considered.

\section{ACKNOWLEDGEMENT}

The support of Petros Aristidou, Ph.D. student at the Univ. of Liège, in implementing the proposed controller in RAMSES is gratefully acknowledged.

\section{REFERENCES}

[1] M.J. Dolan, E.M. Davidson, I. Kockar, G.W. Ault and S.D.J. McArthur, "Distribution power flow management utilizing an online optimal power flow technique," IEEE Transactions on Power Systems, vol. 27, no. 2. pp. 790-799, May 2012.

[2] A.G. Madureira and J.A. Pecas Lopes, "Coordinated voltage support in distribution networks with distributed generation and microgrids," IET Renewable Power Generation, vol. 3, no. 4, pp. 439-454, Dec. 2009.

[3] ENTSO-E, "Network code on Demand Connection" pp. 1-63, Dec. 2012. [Online]. Available: https://www.entsoe.eu.

[4] A. Keane, L.F. Ochoa, E. Vittal, C.J. Dent and G.P. Harrison, "Enhanced utilization of voltage control resources with distributed generation," IEEE Transactions on Power Systems, vol. 26, no. 1, pp. 252-260, Feb. 2011

[5] L.F. Ochoa, A. Keane and G. Harrison, "Minimizing the reactive support for distributed generation: enhanced passive operation and smart distribution networks," IEEE Transactions on Power Systems, vol. 26, no. 4, pp. 2134 - 2142, Nov. 2011.

[6] M. E. Baran and I. M. El-Markabi, "A multiagent-based dispatching scheme for distributed generators for voltage support on distribution feeders," IEEE Transactions on Power Systems, vol. 22, no. 1, pp. 52-59, Feb. 2007.

[7] A. Borghetti, M. Bosetti, S. Grillo, S. Massucco, C.A. Nucci, M. Paolone, and F. Silvestro, "Short-term scheduling and control of active distribution systems with high penetration of renewable resources," IEEE Systems Journal, vol. 4, no. 3, pp. 313-322, Sept. 2010.

[8] E. Riva Sanseverino, M.L. Di Silvestre, M.G. Ippolito, A. De Paola and G. Lo Re, "An execution, monitoring and replanning approach for optimal energy management in microgrids," Energy, vol. 36, no. 5, pp. 3429-3436, May 2011.

[9] M. Biserica, B. Berseneff, Y. Besanger, and C. Kieny, "Upgraded coordinated voltage control for distribution systems," IEEE PowerTech, Trondheim, pp.1-6, Jun. 2011.

[10] J. M. Maciejowski, Predictive Control With Constraints. Englewood Cliffs, NJ: Prentice-Hall, 2002.

[11] G. Valverde and T. Van Cutsem, "Model Predictive Control of Voltages in Active Distribution Networks," accepted for publication in IEEE Transactions on Smart Grid, Special Issue on Optimization Methods and Algorithms Applied to Smart Grid, to appear in 2013.

[12] United Kingdom Generic Distribution Network (UKGDS). [Online]. Available: http://sedg.ac.uk.

[13] D. Fabozzi, "Decomposition, Localization and Time-Averaging Approaches in Large-Scale Power System Dynamic Simulation, Ph.D. dissertation, University of Liège, 2012. [Online]. Available: http://hdl.handle.net/2268/126720.

[14] A collection of Fortran codes for large scale scientific computation, 2011. [Online]. Available: http://www.hsl.rl.ac.uk. 